\title{
Preface to the Special Issue on Immersive Environments: Challenges, Research and New Developments
}

\author{
Anasol Peña-Rios ${ }^{1, *}$, Johanna Pirker ${ }^{2}$, Christian Gütl $^{2}$ and Michael Gardner ${ }^{1}$ \\ ${ }^{1}$ University of Essex, UK \\ ${ }^{2}$ Graz University of Technology, Austria
}

Received on 6 June 2016, accepted on 7 June 2016, published on 27 June 2016

Copyright (c) 2016 A. Peña-Rios et al., licensed to EAI. This is an open access article distributed under the terms of the Creative Commons Attribution licence (http://creativecommons.org/licenses/by/3.0/), which permits unlimited use, distribution and reproduction in any medium so long as the original work is properly cited.

doi: 10.4108/eai.27-6-2016.151520

\section{Preface}

Immersion could be defined as a subjective impression of participating in a comprehensive, realistic experience [1]. However, immersion should not be treated as a unique property, as it is achieved from a complex interaction of representational fidelity and learner interaction, holding a dependency on other aspects of the environment $[2,3]$. The use of immersive environments which create a feeling of 'presence' naturally allows for more complex social interactions and designed experiences [4]. A particular use of these technologies is in educational settings, where they can enhance learning experiences, foster participation, collaboration, creativity and engagement; creating huge opportunities for integration and research. Effective immersive learning experiences can be created with multiple media using myriad techniques and employing a wealth of knowledge that spans many disciplines. This includes but is not limited to computer science, user experience and media design, the learning sciences, architecture, game development, artificial intelligence, biology, medicine, and thousands of disciplinary and occupational content areas wherein immersive learning and training may be relevant.

The Immersive Learning Research Network (iLRN) is " $a$ n international organization of developers, educators, and research professionals collaborating to develop the scientific, technical, and applied potential of immersive learning" [5]. The vision of the network is to develop a comprehensive research and outreach agenda that encompasses the breadth and scope of learning potentialities, affordances and challenges of immersive learning environments. The first international conference
iLRN 2015 held in Prague, Czech Republic, attracted a number of high-quality contributions. As a follow-up, this special issue was organised as an open call to seek a wider set of contributions from the research community, including extended versions of iLRN 2015 best papers.

\section{Contributions included in the Special Issue}

The articles contributed to this Special Issue present a sample of diverse technologies used by educators and researchers in the creation of immersive environments. Moreover, they illustrate some of the multiple opportunities for their use within the learning environment, regardless of the topic; to illustrate this, the special issue presents case studies applied to a variety of fields including politics, law and medicine.

In Expert evaluation of the Westminster Serious Games Platform (wmin-SGP), a tool for dynamic creation of roleplaying simulations, based on usability heuristics and the Octalysis gamification framework, D. Economou, I. Doumanis, F. Pedersen, P. Kathrani, M. Mentzelopoulos, V. Bouki and N. Georgalas introduce a platform for the dynamic creation of bespoke 3D virtual scenarios for roleplay based learning; which include embodied virtual humans for verbal and non-verbal interaction; incorporating elements of gamification. The dynamic creation of roleplay simulations enables the use of this platform for educators and domain experts without the need of having technical skills in games design or development. To illustrate this, the authors present two case studies of the use of their platform in two different topics: Politics and Law, focusing on usability and gamification principles.

*Corresponding author. Email:acpena@ essex.ac.uk 
In OSCase: Data Schemes, Architecture and Implementation details of Virtual Patient repurposing in Multi User Virtual Environments, P. Antoniou, L. Ioannidis and P. Bamidis introduce us to medical training based on experiential learning using Virtual Patients (VP). Here, the authors present a platform for transferring existing web-based VPs simulations to multi-user virtual environments (MUVEs), using web-based models created with OpenLabyrinth (OLab), and transferring them to 3D virtual representations in OpenSimulator (OpenSim) with the aim of exploiting the immersion and engagement potential of 3D virtual learning environments, and enhancing the medical training provided.

In The use of a cyber campus to support students experiencing barriers accessing education, L. Nisiotis, M. Beer and E. Uruchurtu present a case study of the use of a $3 \mathrm{D}$ virtual university campus as a tool to support and enhance distance learning, bringing forth benefits of 3D virtual learning environments for remote students, and identifying characteristics that contribute to students' online learning experiences based on a user study.

In Collaboration in 3D Virtual Worlds: a protocol for case study research, A. Cruz, H. Paredes, B. Fonseca, P. Martins and L. Morgado, presents us with a protocol for research of presence and cooperation in virtual worlds, analysing how nonverbal communication influences collaboration in three-dimensional virtual worlds. Within this collaboration, the authors present the analysis of two cases using the proposed protocol.

In Observing Coaching and Reflecting: Metalogue - A Multi-modal Tutoring System with Metacognitive Abilities, J. Van Helvert, P. Van Rosmalen, D. Borner, V. Petukhova, J. Alexandersson, H. Weerd, N. Taatgen and C. Stevens, present the Metalogue project, which aims to deliver a multi-modal, multi-party dialogue system able to evaluate and give feedback about human interaction, applied to debate and negotiation scenarios. The paper provides detail of the system-generated learner feedback provided (inperformance and post-performance) to support the pedagogical framework in which user interactions will take place. Within this contribution, the authors discuss implementation issues, pilots and the key challenges that this project had to overcome.

In Smart Ambient Learning with Physical Artifacts Using Wearable Technologies, I. Koren and R. Klamma, propose a multicontextual framework to integrate concepts for teaching and learning using wearable technologies. The paper presents a case study based on an exhibition as an educational experience, focusing on a multi-modal experience based on physical artifacts, which can commence before the user's physical presence at the exhibition, and continue after it, integrating community learning analytics for self-reflection.
We believe this special issue provides us with a timely opportunity to discuss opportunities for research in this strategically important area, presenting an exciting window to the challenges and new developments that immersive environments pose, as exemplified by the peer-reviewed papers presented.

\section{Acknowledgements}

The guest editors of this Special Issue of EAI Endorsed Transactions on Future Intelligent Educational Environments would like to acknowledge the reviewers for their invaluable help in the assessment of the submissions. Additionally, we would like to thank the FIEE chief editors and Lucia Kisova (EAI) for her advice on technical issues. Finally, we would like to thank the authors who contributed with their research work in the creation of this Special Issue on Immersive Environments: Challenges, Research and New Developments.

\section{References}

[1] Dede, C. (2009). Immersive interfaces for engagement and learning. Science, 323(5910), 66-69.

[2] B. Dalgarno and M. J. W. Lee. (2010) What are the learning affordances of 3-D virtual environments?. British Journal of Educational Technology, 41(1):10-32.

[3] J. Hedberg and S. Alexander. (1994) Virtual Reality in Education: Defining Researchable Issues. Educational Media International, 31(4):214-220.

[4] De Freitas, S., Rebolledo-Mendez, G., Liarokapis, F., Magoulas, G., \& Poulovassilis, A. (2010). Learning as immersive experiences: Using the four-dimensional framework for designing and evaluating immersive learning experiences in a virtual world. British Journal of Educational Technology, 41(1), 69-85.

[5] Immersive Learning Research Network (2016). About us Immersive Learning Research Network. [ONLINE] Available at: https://immersivelrn.org/about-us/what-isilrn/. [Accessed 26 April 2016]. 